\title{
Efficacy of Slow Rate Ventriculolumbar Perfusion Chemotherapy for Leptomeningeal Carcinomatosis: Interim Result of a Phase II Study
}

\author{
Young Hoon Choi', Ho-Shin Gwak², Jungnam Joo ${ }^{3}$, Ji-Woong Kwon4, Sang-Hoon Shin' ${ }^{4}$, Heon Yoo ${ }^{4}$, Ji-Hye Lee ${ }^{4}$, Ji-Hye Youn ${ }^{4}$ \\ 'Departments of Neurosurgery, Seoul National University Hospital, Seoul, Korea \\ 2Department of Cancer Control, Graduate School of Cancer Science and Policy, National Cancer Center, Goyang, Korea \\ ${ }^{3}$ Biometric Research Branch, National Cancer Center, Goyang, Korea \\ ${ }^{4}$ Neuro-Oncology Clinic, National Cancer Center, Goyang, Korea
}

Received January 14, 2019

Revised March 29, 2019

Accepted June 24, 2019

\section{Correspondence}

Ho-Shin Gwak

Department of Cancer Control, Graduate School of Cancer Science and Policy, National Cancer Center, 323 Ilsan-ro, llsandong-gu,

Goyang 10408, Korea

Tel: +82-31-920-1666

Fax: +82-31-920-2798

E-mail: nsghs@ncc.re.kr
Background To evaluate the efficacy of modified ventriculolumbar perfusion (VLP) chemotherapy with methotrexate on leptomeningeal carcinomatosis in terms of symptomatic response and side effects.

Methods Previous infusion rate of $20 \mathrm{~mL} / \mathrm{h}$ was reduced to $15 \mathrm{~mL} / \mathrm{h}$ for the purpose of decreasing constitutional side effects of VLP such as nausea/vomiting, insomnia and confusion. The primary outcome was the response rate of increased intracranial pressure (ICP), and the secondary outcome was the occurrence of side effects compared to previous $20 \mathrm{~mL} / \mathrm{h}$ trial. This interim analysis to validate the reduced infusion rate is not to affect the original effect of VLP chemotherapy.

Results All forty-seven patients were enrolled including 22 patients with increased ICP. Thirteen patients out of these (59\%) got normalized ICP after VLP chemotherapy. Moderate to severe (grade $2-3)$ confusion was observed in 3 patients (6\%) and it was significantly reduced compared to those (23\%) in the VLP $20 \mathrm{~mL} / \mathrm{h}$ ( $p=0.017)$. Grade $2-3$ nausea/vomiting was also reduced from $64 \%$ to $45 \%$ but failed to reach statistical significance $(p=0.08)$. Median overall survival (OS) was 5.3 months $(95 \%$ confidence interval, 3.55-7.05) and patients OS, who received maintenance VLP was significantly prolonged compared to patients who underwent induction VLP only (5.8 vs. 3.4 months, $p=0.025$ ).

Conclusion VLP of reduced perfusion rate $(15 \mathrm{~mL} / \mathrm{h})$ showed compatible control rate of increased ICP at this interim analysis. Decreased moderate to severe side effects and prolonged OS in patients received maintenance VLP encourage us to evaluate the effectiveness of this trial further.

Key Words Intraventricular infusion; Chemotherapy; Leptomeningeal carcinomatosis; Methotrexate; Perfusion.

\section{INTRODUCTION}

Leptomeningeal carcinomatosis (LMC) is one of the most formidable, incurable, terminal-stage, central nervous system (CNS) solid tumor diseases, and is devastating to patient function. Intra-cerebrospinal fluid (CSF) chemotherapy has resulted in marginal survival prolongation of 4-9 months [1-4].

\footnotetext{
This is an Open Access article distributed under the terms of the Creative Commons Attribution Non-Commercial License (https://creativecommons.org/licenses/by-nc/4.0) which permits unrestricted non-commercial use, distribution, and reproduction in any medium, provided the original work is properly cited.

Copyright $\odot 2019$ The Korean Brain Tumor Society, The Korean Society for NeuroOncology, and The Korean Society for Pediatric Neuro-Oncology
}

However, the effectiveness of this therapeutic modality is greatly hindered by CSF flow disturbance, which has been known to prevalent up to $50 \%$ of patients with LMC [5-7]. This CSF flow disturbance, is manifested as headache, increased intracranial pressure (ICP), and hydrocephalus, resulting in uneven distribution of the intraventricularly injected drug, and might cause encephalopathy through a transependymal drug penetration to brain parenchyma [8-12].

The potential benefit of intraventricular drug infusion has been proposed to achieve drug distribution throughout brain parenchyma and CSF space, even under conditions of disturbed CSF flow [13,14]. Nakagawa et al. [15] had tried ven- 
triculolumbar perfusion (VLP) chemotherapy with methotrexate (MTX) and cytosine arabinoside (Ara-C) in 1990s for the purpose of improving lower limb function and decreasing neurotoxicity by enforced drug perfusion from the ventricle to thecal sac. They found $50 \%$ of regaining ambulatory function but profound side effects hindered further study.

Previously, we conducted a phase I/II study of VLP chemotherapy with MTX for LMC and found that a perfusion rate of $20 \mathrm{~mL} / \mathrm{h}$ and a total daily MTX dose of $24 \mathrm{mg}$ was tolerable $[16,17]$. In the previous phase II study, ICP was normalized after VLP therapy in 20 patients (65\%) among 31 patients who had increased ICP. However, constitutional side effects of VLP including nausea/vomiting, sleep disturbance, and confusion occurred up to $60 \%$ at grade 2 severity (CTCAE version 3.0) [18]. We reminded that the side effects had occurred after artificial CSF infusion before MTX injection and more severe side effects occurred at higher perfusion rate $(40 \mathrm{~mL} / \mathrm{h})$ in the previous phase I study. Thus, we assume that reduced perfusion rate $(15 \mathrm{~mL} / \mathrm{h})$ might decrease these constitutional side effects and provide more comfort treatment to patients with LMC. The primary outcome was the response rate of increased ICP, and the secondary outcome was the occurrence of moderate to severe (grade 2-3) side effects compared to those of perfusion rate of $20 \mathrm{~mL} / \mathrm{h}$.

\section{MATERIALS AND METHODS}

All patients provided written informed consent prior to enrollment in this phase II clinical trial. The protocol of this clinical trial was reviewed in accordance with the precepts established by the Helsinki Declaration and approved by the Institutional Review Board of the National Cancer Center of Korea (NCCCTS-10-511). This clinical trial was registered with the government-owned official site (http://cris.nih. go.kr) before patient enrollment (KCT0000082).

\section{Eligibility criteria}

Inclusion criterion was patient: 1) LMC diagnosis by either positive CSF cytology or definitive MRI findings [19], 2) had at least one of the following LMC related symptoms of increased ICP $\left(>20 \mathrm{~cm} \mathrm{H}_{2} \mathrm{O}\right)$, altered mentality due to hydrocephalus, cauda equina symptoms or cranial neuropathy. Exclusion criteria were: 1) CSF pathway block on either whole spine MRI or radioisotope cisternography, 2) space-occupying brain lesion that might cause herniation, 3) hemorrhagic CNS metastases, 4 ) $\geq$ grade- 3 leucopenia ( $\mathrm{WBC}<2,000$ or ANC $<1,000 / \mathrm{mm}^{3}$ ) or thrombocytopenia $(<50 \mathrm{k})$.

\section{VLP procedure}

The VLP procedures were basically the same as those used in the previous phase II study except for reduced perfusion rate [17]. Briefly, a ventricular catheter was installed stereotactically and connected to subgaleal reservoir of either Chemoport (Celsite ${ }^{\circledR}$, ST205, B. Braun, Boulogne Cedex, France) [20] or V-port (INSUNG Medical Co., Ltd, Wonju, Korea) [21]. Artificial CSF that was premixed with MTX was perfused at $15 \mathrm{~mL} / \mathrm{h}$ by an infusion pump with a warming kit $\left(37.5^{\circ} \mathrm{C}\right)$ via designated 21-gauge hooked needle inserted and maintained in position with a sterile closed dressing. A lumbar drainage catheter was inserted into thecal sac at the time of VLP and CSF drainage was achieved by hydrostatic pressure. The lumbar drainage rate was checked on an hourly basis and the drainage set height was adjusted so that the drainage rate equaled the infusion rate.

\section{Treatment protocol and drug level monitoring}

Different from previous phase II study of two consecutive continuous infusion over 72 hours 3 days apart (so-called 'induction'), we regulated VLP 'maintenance' cycle of a VLP over 72 hours at every 28 days until patients experienced progression or refused to undergo further chemotherapy. The schematic of treatment protocol is illustrated in Fig. 1.

CSF samples were taken every morning on day 1-3 to ensure the MTX plateau level was reached and to monitor possible CSF infection as early as possible. Serum MTX concentration was also checked daily. If the MTX serum concentration was above $0.15 \mu \mathrm{M}$, a $30 \mathrm{mg}$ leucovorin rescue was administered i.v. every 6 hours.

\section{Response evaluation}

Pre-VLP ICP was defined as the highest ICP among related procedure of the reservoir installation, lumbar puncture or the needle insertion at reservoir on VLP. If ICP was equal or above $20 \mathrm{~cm} \mathrm{H}_{2} \mathrm{O}$, we consider the patients had increased ICP. Post-VLP ICP was measured on the last day of VLP induction (day 14) 6 hours after the last VLP and before lumbar drainage removal. Normalization $\left(<20 \mathrm{~cm} \mathrm{H}_{2} \mathrm{O}\right)$ of increased ICP was counted as the response.

\section{Toxicity evaluation}

The targeted VLP side effects included nausea/vomiting, sleep disturbance, and encephalopathy (confusion) were closely monitored and recorded daily. Side effects were assessed using the Common Terminology Criteria for Adverse Events (CTCAE, version 3.0) [18]. The most severe grade during the VLP chemotherapy represents the grade of side effects of the patient.

\section{Statistical analysis}

The necessary patient number for this phase II study was 


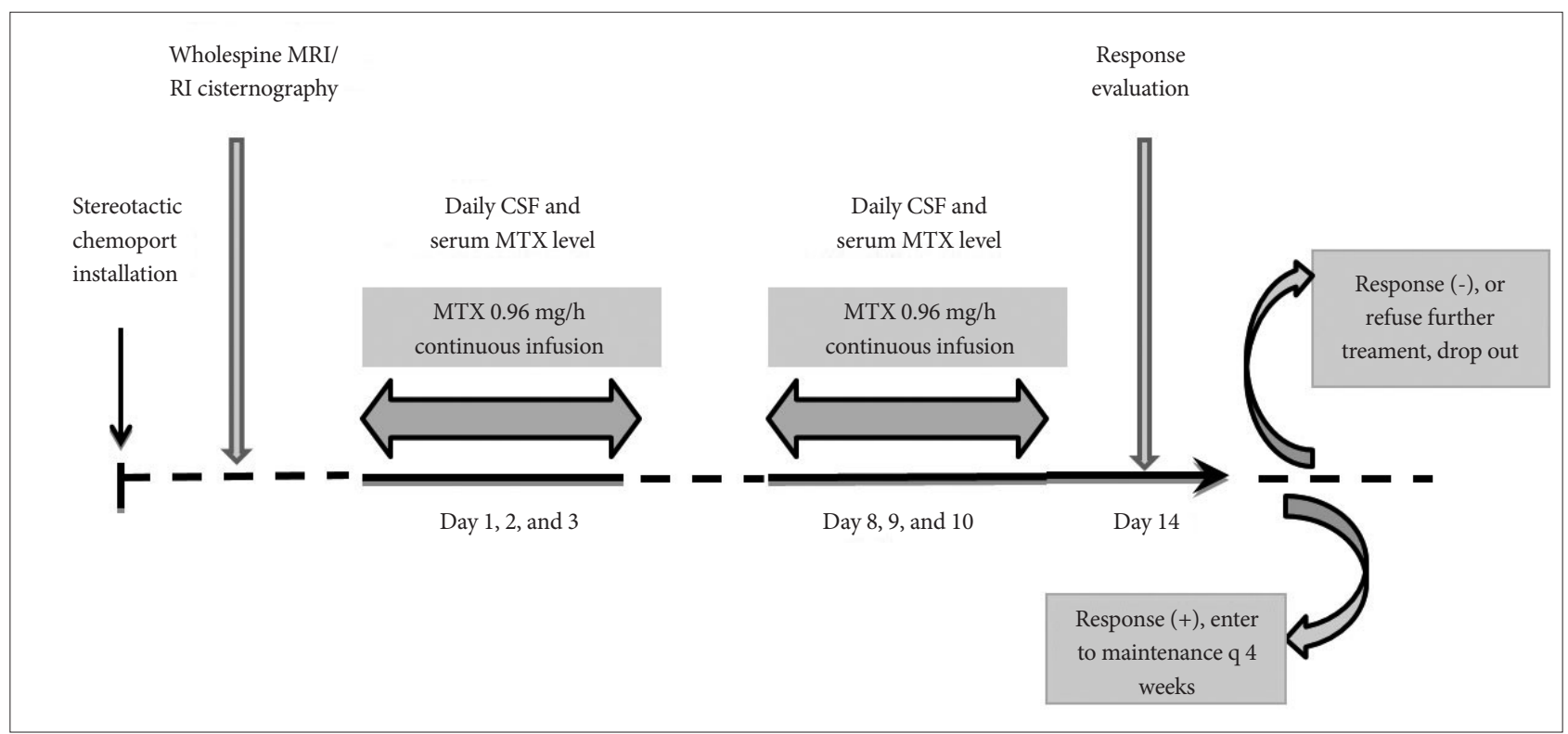

Fig. 1. Treatment schema of ventriculolumbar MTX perfusion chemotherapy. MTX, methotrexate; CSF, cerebrospinal fluid; RI, radioisotope.

calculated using Simon's minimax two-stage design [22]. The primary endpoint was the response rate of increased ICP, which was available for comparison from previous phase II VLP trial [17]. A review of historical data suggested a null response rate (p0) of $50 \%$ compared to interesting response rate (p1) of previous phase II. Thus, assuming uninteresting response rate for early termination $50 \%$ (p0), 20\% of power and 0.1 of one-sided type-I error, the number of patients required at stage-one was 22 . If 10 or fewer responses were obtained at stage I, the study will stop. Otherwise, 28 additional patients were to be included at stage-2, for total of 50 patients.

Comparison of moderate to severe side effects between this VLP of $15 \mathrm{~mL} / \mathrm{h}$ and previous $20 \mathrm{~mL} / \mathrm{h}$ was done using chisquare test with continuity correction. Overall survival data were defined as the time from LMC diagnosis to the end of the observation period or patient death, whichever occurred first. The survival rates were estimated using the Kaplan-Meier method and the differences of survival between the investigated factors were compared using the log-rank test. We considered a $p$ value less than 0.05 to indicate a statistically significant difference. All statistical analyses were performed using SPSS (version 18.0, SPSS Inc., Chicago, IL, USA).

\section{RESULTS}

\section{Patients and VLP chemotherapy}

From May 2014 to November 2016, a consecutive 48 patients were analyzed until 22 patients with increased ICP for interim analysis was enrolled and 47 patients were evaluated as one patient withdrew the informed consent.
Table 1. Clinical characteristics of the patients who underwent ventriculolumbar perfusion chemotherapy

\begin{tabular}{lc}
\hline \multicolumn{1}{c}{ Characteristics } & No. of patient $(\mathrm{n}=47)$ \\
\hline Gender (male/female) & $25 / 22$ \\
Age $(\mathrm{yr})$ & $59(38-82)$ \\
Primary cancer & \\
Non-small cell lung cancer & $35(74)$ \\
Breast cancer & $7(15)$ \\
Others & $5(11)$ \\
$\quad$ Melanoma & 1 \\
$\quad$ Ovarian cancer & 1 \\
$\quad$ Small cell lung cancer & 1 \\
$\quad$ PXA & 1 \\
MUO & 1 \\
KPS score & $70(30-100)$ \\
$\geq 70$ & $30(64)$ \\
$<70$ & $17(36)$ \\
\hline
\end{tabular}

Data are presented as median (range) or number (\%). MUO, malignancy of unknown origin; PXA, pleomorphic xanthoastrocytoma, KPS: Karnofsky Performance Status

Twenty-five patients were female and 22 patients were male (Table 1). The median patient age was 59 years (range $38-82$ years). Thirty-five patients (74\%) had non-small cell lung cancer (NSCLC), and seven patients had breast cancer. The remaining five patients presented with melanoma, ovarian cancer, small cell lung cancer, glioma and adenocarcinoma of unknown primary origin, respectively. The median Karnofsky Performance Status (KPS) score at the time of enrollment was 70 (range 30-100), and 17 patients (36\%) were below KPS 70 (KPS=60 in 4 patients, KPS=50 in 10 patients, 
$\mathrm{KPS}=40$ in 2 patients, and KPS=30 in 1 patient). Among these, 42 patients (89\%) completed 2 consecutive infusion of VLP 'induction' chemotherapy while eight patients dropped out due to: progression of primary cancer and LMC each, patient's intolerance to side effects, unexpected thrombocytopenia $(<50 \mathrm{k})$, treatment related meningitis and Herpes zoster infection. Thirty-four patients $(72 \%)$ received 'maintenance' VLP chemotherapy of median 3 rounds (range 1-10).

\section{Response of increased ICP}

In this interim analysis, 22 out of 47 patients (47\%) had increased ICP before VLP (Fig. 2). The ICP was normalized in 13 out of 22 patients (59\%), and the response rate was higher than uninteresting rate $(\mathrm{p} 0=50 \%)$. However, three patients with normal ICP before VLP induction showed increased ICP after VLP. The ICP before VLP was median $18 \mathrm{~cm} \mathrm{H}_{2} \mathrm{O}$ (range 3-70) in all patients and the ICP after VLP was medi-

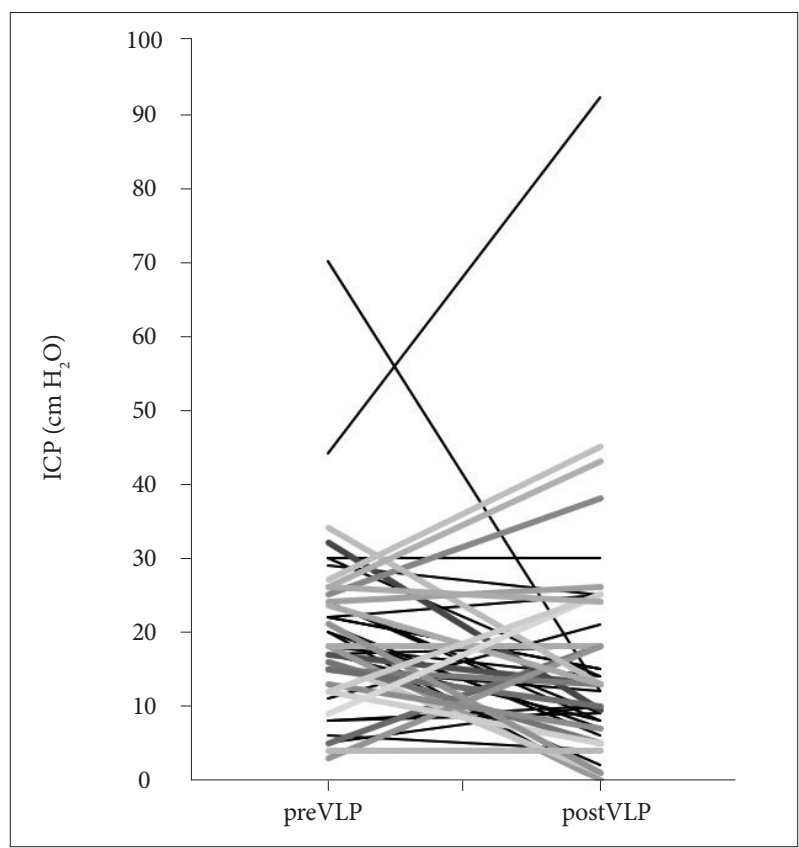

Fig. 2. Distribution of ICP. The distribution of individual patient's ICP $\left(\mathrm{cm} \mathrm{H}_{2} \mathrm{O}\right)$ is shown before (left) and after (right) VLP chemotherapy with methotrexate. Gray scales differentiate individual patients. ICP, intracranial pressure; VLP, ventriculolumbar perfusion.

Table 2. 'Constitutional' side effects related to ventriculolumbar perfusion chemotherapy $(n=47)$

\begin{tabular}{cccc}
\hline Grade* $^{*}$ & Nausea/vomiting & Sleep disturbance & Confusion \\
\hline 0 & $13(28)$ & $29(62)$ & $39(83)$ \\
1 & $13(28)$ & $13(28)$ & $5(11)$ \\
2 & $17(36)$ & $5(11)$ & $1(2)$ \\
3 & $4(9)$ & - & $2(4)$ \\
\hline
\end{tabular}

Numbers in parenthesis are vertical proportion. *Grade is based on CTCAE version 3.0 [18] (see Methods section) an $13 \mathrm{~cm} \mathrm{H}_{2} \mathrm{O}$ (range 0-92), and the ICP was significantly reduced after VLP ( $p<0.01$, Wilcoxon singed rank test).

\section{Side effects of VLP therapy}

'Constitutional' VLP side effects such as nausea/vomiting, sleep disturbance, and confusion were graded with the CTCAE scale [18] (Table 2). Thirteen patients (28\%) described that they had no additional nausea/vomiting on VLP chemotherapy, but another 13 patients experienced mild grade 1 nausea/vomiting, for which treatment is not necessary. Seventeen patients (36\%) suffered from grade 2 nausea/vomiting and the remaining 4 patients could not eat any meal during the VLP (grade 3). Sleep disturbance was observed in 18 patients (38\%) but 13 of those patients did not want to have sleeping pills (grade 1) to fall asleep. Confusion as a manifestation of encephalopathy was observed in 8 patients (17\%) and 2 of them had a difficulties in communication (grade 3) during the VLP. To compare the occurrence of side effects in VLP $15 \mathrm{~mL} / \mathrm{h}$ to VLP $20 \mathrm{~mL} / \mathrm{h}$, we grouped side effects into nil to mild (grade $0-1$ ) and moderate to severe (grade 2-3) side effects. In VLP $15 \mathrm{~mL} / \mathrm{h}$, nausea/vomiting occurred as grade $0-1$ in 26 patients (56\%) and as grade $2-3$ in 21 patients (45\%) (Fig. 3A). The occurrence of moderate to severe nausea/vomiting showed a tendency to decrease compared to 41 patients (64\%) in VLP $20 \mathrm{~mL} / \mathrm{h}$ ( $p=0.053)$. Grade 2-3 sleep disturbance was observed in 6 patients (11\%) in VLP $15 \mathrm{~mL} /$ $\mathrm{h}$ and it was not significantly different from 6 patients (9\%) in VLP $20 \mathrm{~mL} / \mathrm{h}$ (Fig. 3B). Moderate to severe confusion was recorded in 3 patients (6\%) in VLP $15 \mathrm{~mL} / \mathrm{h}$ and those rate was significantly reduced compared to 15 patients (23\%) in VLP $20 \mathrm{~mL} / \mathrm{h}$ (Fig. 3C, $p=0.017$ ).

Seizure as another possible manifestation of encephalopathy was observed in 4 patients but the episode was controlled with administration of short-acting sedatives lorazepam (Ativan ${ }^{\circledR}$ ) and anti-epileptics except for one patient who already had a seizure before VLP (grade 3).

CSF-infection occurred in 3 patients during and after VLP induction. All patients undergone removal of infected reservoir and catheter, and two of them had extraventricular drainage until clearance of infection, then new device was inserted. One patient refused to receive further treatment and received ventriculoperitoneal shunt insertion then transferred to hospice care. Another one patient after controlled infection received Ommaya reservoir re-insertion and done VLP induction. But because of systemic cancer progression, we interrupted futher VLP. Last one patient expired due to septic shock related CSF infection. As our protocol include hematologic abnormalities of equal or less than grade 2 (CTCAE version 3.0), grade 1 or 2 cytopenia was not automatically noticed by protocol per se. In detail, 

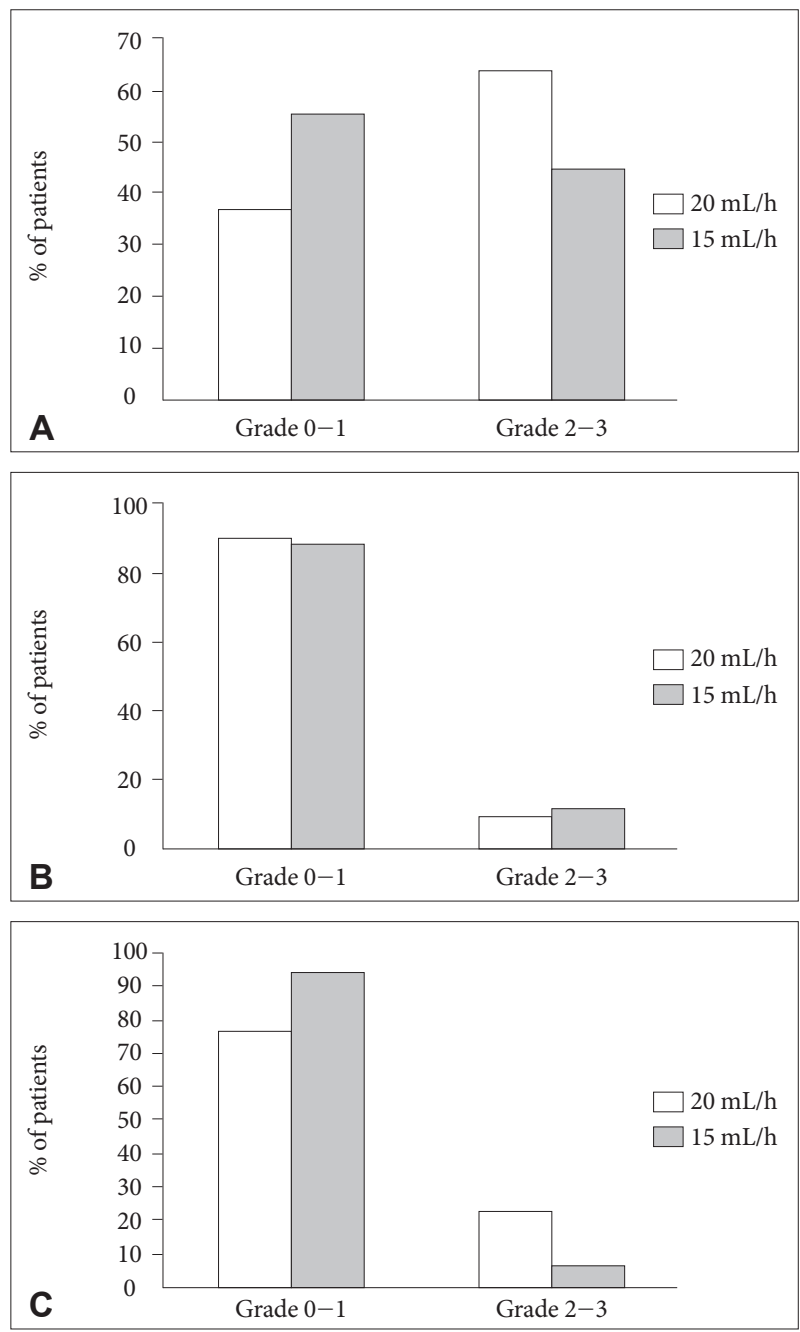

Fig. 3. Side effects during ventriculolumbar perfusion chemotherapy comparison of perfusion rate between $15 \mathrm{~mL} / \mathrm{h}$ and $20 \mathrm{~mL} / \mathrm{h}$. A: Nausea and vomiting, moderate to severe Grade 2-3 $(p=0.053)$. B: Sleep disturbance, moderate to severe Grade 2-3 $(p=0.805)$. C: Confusion, moderate to severe Grade 2-3 $(p=0.017)$.

grade 3 anemia was not observed, and grade 2 anemia was recorded in 4 patients (8.5\%), all those patients were previously heavily treated with cytotoxic chemotherapy. Grade 2 leucopenia occurred in other 3 patients (6.4\%), and grade 3 leucopenia was happened in one patient (2.1\%). All these patients were resolved without any further treatment, and had a history of cytotoxic chemotherapy before enrolling into this study except one patient only received receptor tyrosine kinase inhibitor gefitinib $\left(\right.$ Iressa $\left.^{\circledR}\right)$. Only one patient with grade 4 leucopenia (ANC $<500$ cells $/ \mathrm{mm}^{3}$ ) had a granulocyte stimulating factor injection, and he had received more than 3 years of cytotoxic chemotherapy including cisplatin, docetaxel, gemcitabine, irinotecan and pemetrexed. Grade 3 thrombocytopenia $(<50 \mathrm{k})$ occurred in 2 patients (4.3\%), and they also had received cytotoxic chemotherapy heavily prior to VLP chemotherapy. One of them had a platelet transfusion.

\section{Overall patient survival}

All patients had expired at the time of analysis. The median overall survival (OS) of 47 patients was 5.3 months (95\% confidence interval, 3.55-7.05) (Fig. 4A). Thirteen patients who received 'induction' VLP chemotherapy only showed significantly shorter median OS compared to 34 patients who underwent 'maintenance' VLP chemotherapy (3.4 vs. 5.8 months, $p=0.025$ ) (Fig. 4B).

\section{DISCUSSION}

\section{ICP control rate in VLP chemotherapy}

The improvement of increased ICP was not scientifically described in previous studies from literature. In our previous study of patients with LMC from NSCLC treated by intraventricular chemotherapy, 20/69 patients (29\%) with increased ICP $\left(>15 \mathrm{~cm} \mathrm{H}_{2} \mathrm{O}\right)$ achieved normal ICP [23], whereas in the previous phase II clinical trial, $31 / 41$ patients $(76 \%)$ with increased ICP at the start of VLP were normalized. The ICP control rate was one of advantages of VLP over conventional intraventricular chemotherapy and has encouraged us to perform VLP to patients having CSF flow disturbance from LMC. As a result, the primary end point of this reduced perfusion rate trial was not the decreased side effects but the 'non-inferior' response rate of increased ICP.

\section{Prolonged OS after VLP chemotherapy}

It is established that factors such as patient performance, presenting symptoms, systemic disease status, increased ICP, and primary cancer type affect survival of patients with LMC receiving intraventricular chemotherapy [1,3,23-26]. We had compared the survival between patients treated with VLP and those treated with conventional intraventricular chemotherapy for LMC from the same primary cancer of NSCLC in the previous study. In comparison, VLP treatment significantly doubled patient survival from a median 89 days with conventional intraventricular chemotherapy to 187 days with VLP. In this interim result of NSCLC as a major primary cancer (75\%), we evaluated the patients OS of 5.3 months, which can be comparable to previous phase II study. To evaluate the effectiveness of continued VLP chemotherapy, we introduced 'maintenance' VLP chemotherapy every 4 weeks after 'induction' VLP chemotherapy, and it significantly prolonged OS compared to that of patients had 'induction' chemotherapy only. However, we should prove this effectiveness by prospective controlled study as generally patients in poor medical/general condition tend to quit further chemotherapy. 

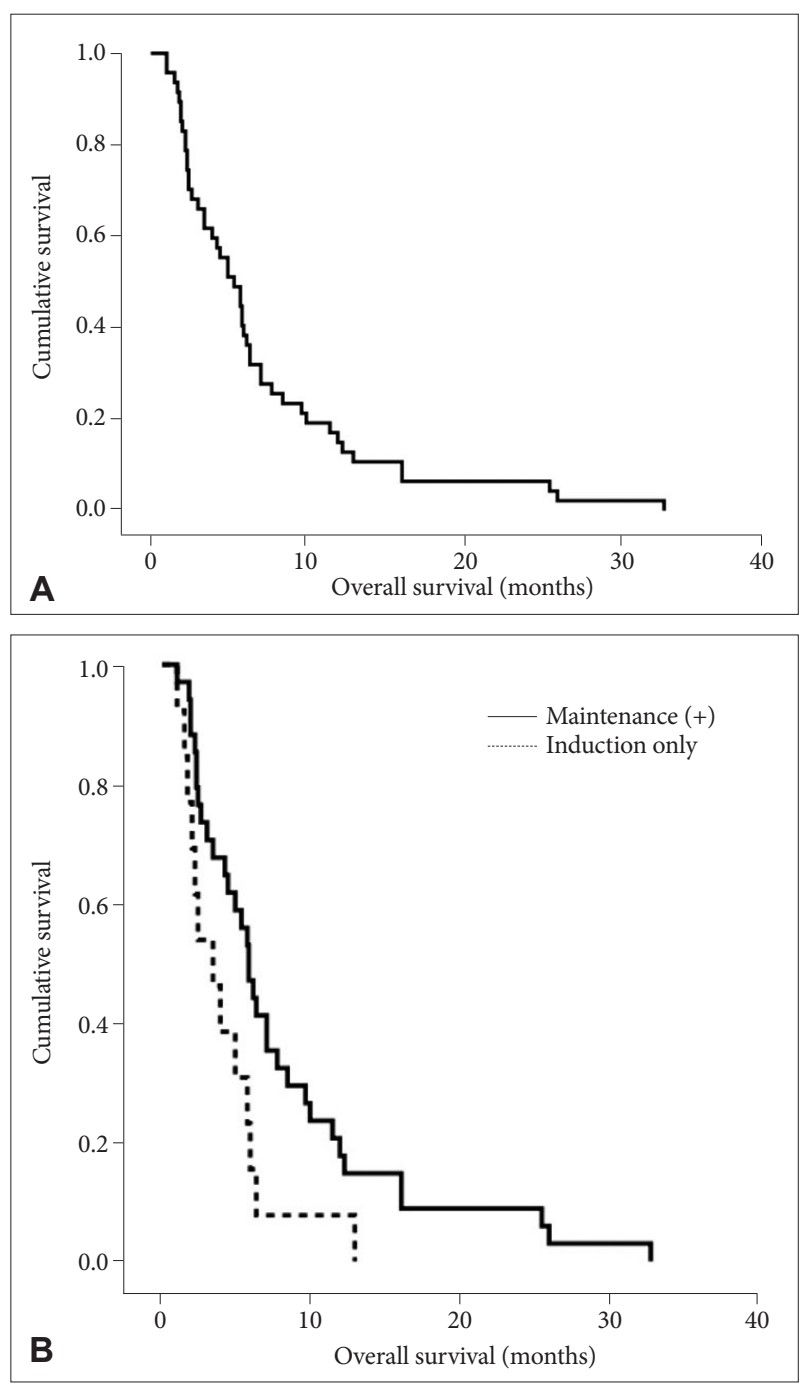

Fig. 4. Kaplan-Meier overall survival curve in VLP $15 \mathrm{~mL} / \mathrm{h}$ of $(A)$ all 47 patients who were 'intention-to-treat' with VLP, (B) comparison of overall survival of patients who received maintenance VLP chemotherapy versus patients who underwent induction VLP chemotherapy only $(p=0.025)$. VLP, ventriculolumbar perfusion.

\section{Decreased side effects of VLP chemotherapy in reduced perfusion rate}

The improvement of VLP technique in our study was reduced perfusion rate while maintaining its effectiveness. During our phase I study, we noticed that patients complained of nausea/vomiting and sleep disturbance during artificial CSF perfusion before bolus MTX injection, and more severe side effect at $40 \mathrm{~mL} / \mathrm{h}$ perfusion [16].

Side effects of confusion in reduced perfusion rate of 15 $\mathrm{mL} / \mathrm{h}$ were decreased significantly in comparison with results from perfusion rate of $20 \mathrm{~mL} / \mathrm{h}$, and so the nausea/ vomiting despite statistical insignificance. Sleep disturbance of VLP chemotherapy can be attributable to disruption of 'melatonin surge' for induction of sleep [27]. We could pos- tulate reduced perfusion rate of $15 \mathrm{~mL} / \mathrm{h}$ still disrupt melatonin-surge compared to perfusion rate of $20 \mathrm{~mL} / \mathrm{h}$. Future study measuring CSF melatonin level timely will answer our assumption of VLP-induced sleep disturbance.

\section{Limitations}

Due to difference of patient's characteristics between perfusion rate of $20 \mathrm{~mL} / \mathrm{h}$ VLP and reduced perfusion rate $(15 \mathrm{~mL} /$ h), there are limitations in interpreting results of this study.

\section{Conclusion}

VLP of reduced perfusion rate $(15 \mathrm{~mL} / \mathrm{h})$ showed compatible control rate of increased ICP at this interim analysis. Decreased moderate to severe side effects and prolonged OS in patients received maintenance VLP encourage us to evaluate the effectiveness of this trial further.

If both ICP control rate and OS in reduced perfusion in comparison to VLP $(20 \mathrm{~mL} / \mathrm{h})$ is no statistically difference in future study, reduced perfusion rate VLP could be treatment choice for LMC.

\section{Conflicts of Interest}

The authors have no potential conflicts of interest.

\section{Acknowledgments}

This work was supported by grants from National Cancer Center, Korea (1710871-3), and this research was supported by a grant of the Korea Health Technology R\&D project through the Korea Health Industry Development Institute (KHIDI), funded by the Ministry of Health \& Welfare, Republic of Korea (HI17C1018).

\section{REFERENCES}

1. Wasserstrom WR, Glass JP, Posner JB. Diagnosis and treatment of leptomeningeal metastases from solid tumors: experience with 90 patients. Cancer 1982;49:759-72.

2. Chamberlain MC. Leptomeningeal metastases: a review of evaluation and treatment. J Neurooncol 1998;37:271-84.

3. Chamberlain MC, Kormanik P. Carcinoma meningitis secondary to non-small cell lung cancer: combined modality therapy. Arch Neurol 1998;55:506-12.

4. Sandberg DI, Bilsky MH, Souweidane MM, Bzdil J, Gutin PH. Ommaya reservoirs for the treatment of leptomeningeal metastases. Neurosurgery 2000;47:49-54; discussion 54-5.

5. Grossman SA, Trump DL, Chen DC, Thompson G, Camargo EE. Cerebrospinal fluid flow abnormalities in patients with neoplastic meningitis. An evaluation using 111 indium-DTPA ventriculography. Am J Med 1982;73:641-7.

6. Chamberlain MC. Radioisotope CSF flow studies in leptomeningeal metastases. J Neurooncol 1998;38:135-40.

7. Taillibert S, Laigle-Donadey F, Chodkiewicz C, Sanson M, Hoang-Xuan K, Delattre JY. Leptomeningeal metastases from solid malignancy: a review. J Neurooncol 2005;75:85-99.

8. Price RA, Jamieson PA. The central nervous system in childhood leukemia. II. Subacute leukoencephalopathy. Cancer 1975;35:306-18.

9. Shapiro WR, Young DF, Mehta BM. Methotrexate: distribution in cerebrospinal fluid after intravenous, ventricular and lumbar injections. N Engl J Med 1975;293:161-6.

10. Blasberg RG, Patlak CS, Shapiro WR. Distribution of methotrexate in 
the cerebrospinal fluid and brain after intraventricular administration. Cancer Treat Rep 1977;61:633-41.

11. Bleyer WA, Poplack DG, Simon RM. "Concentration x time" methotrexate via a subcutaneous reservoir: a less toxic regimen for intraventricular chemotherapy of central nervous system neoplasms. Blood 1978;51:835-42.

12. Balis FM, Blaney SM, McCully CL, Bacher JD, Murphy RF, Poplack DG. Methotrexate distribution within the subarachnoid space after intraventricular and intravenous administration. Cancer Chemother Pharmacol 2000;45:259-64.

13. Rubin RC, Ommaya AK, Henderson ES, Bering EA, Rall DP. Cerebrospinal fluid perfusion for central nervous system neoplasms. Neurology 1966;16:680-92.

14. Fleischhack G, Jaehde U, Bode U. Pharmacokinetics following intraventricular administration of chemotherapy in patients with neoplastic meningitis. Clin Pharmacokinet 2005;44:1-31.

15. Nakagawa H, Fujita T, Kubo S, et al. Ventriculolumbar perfusion chemotherapy with methotrexate and cytosine arabinoside for meningeal carcinomatosis: a pilot study in 13 patients. Surg Neurol 1996;45:256-64.

16. Gwak HS, Lim HS, Shin SH, et al. Ventriculolumbar perfusion chemotherapy for the treatment of leptomeningeal carcinomatosis: a phase I study with pharmacokinetic data. Am J Clin Oncol 2013;36:491-9.

17. Gwak HS, Joo J, Shin SH, et al. Ventriculolumbar perfusion chemotherapy with methotrexate for treating leptomeningeal carcinomatosis: a Phase II Study. Oncologist 2014;19:1044-5.

18. Trotti A, Colevas AD, Setser A, et al. CTCAE v3.0: development of a comprehensive grading system for the adverse effects of cancer treatment. Semin Radiat Oncol 2003;13:176-81.
19. Freilich RJ, Krol G, DeAngelis LM. Neuroimaging and cerebrospinal fluid cytology in the diagnosis of leptomeningeal metastasis. Ann Neurol 1995;38:51-7.

20. Gwak HS, Lee CH, Yang HS, et al. Chemoport with a non-collapsible chamber as a replacement for an Ommaya reservoir in the treatment of leptomeningeal carcinomatosis. Acta Neurochir (Wien) 2011;153:1971-8; discussion 1978.

21. Byun YH, Gwak HS, Kwon JW, et al. A novel implantable cerebrospinal fluid reservoir: a pilot study. J Korean Neurosurg Soc 2018;61:640-4.

22. Simon R. Optimal two-stage designs for phase II clinical trials. Control Clin Trials 1989;10:1-10.

23. Gwak HS, Joo J, Kim S, et al. Analysis of treatment outcomes of intraventricular chemotherapy in 105 patients for leptomeningeal carcinomatosis from non-small-cell lung cancer. J Thorac Oncol 2013;8:599-605.

24. Chamberlain MC, Kormanik PA. Prognostic significance of 111indium-DTPA CSF flow studies in leptomeningeal metastases. Neurology 1996;46:1674-7.

25. Bruna J, González L, Miró J, Velasco R, Gil M, Tortosa A; Neuro-Oncology Unit of the Institute of Biomedical Investigation of Bellvitge. Leptomeningeal carcinomatosis: prognostic implications of clinical and cerebrospinal fluid features. Cancer 2009;115:381-9.

26. Park JH, Kim YJ, Lee JO, et al. Clinical outcomes of leptomeningeal metastasis in patients with non-small cell lung cancer in the modern chemotherapy era. Lung Cancer 2012;76:387-92.

27. Debus OM, Lerchl A, Bothe HW, et al. Spontaneous central melatonin secretion and resorption kinetics of exogenous melatonin: a ventricular CSF study. J Pineal Res 2002;33:213-7. 